\title{
Graded Word Sense Assignment
}

\author{
Katrin Erk \\ University of Texas at Austin \\ katrin.erk@mail.utexas.edu
}

\author{
Diana McCarthy \\ University of Sussex \\ dianamesussex.ac.uk
}

\begin{abstract}
Word sense disambiguation is typically phrased as the task of labeling a word in context with the best-fitting sense from a sense inventory such as WordNet. While questions have often been raised over the choice of sense inventory, computational linguists have readily accepted the bestfitting sense methodology despite the fact that the case for discrete sense boundaries is widely disputed by lexical semantics researchers. This paper studies graded word sense assignment, based on a recent dataset of graded word sense annotation.
\end{abstract}

\section{Introduction}

The task of automatically characterizing word meaning in text is typically modeled as word sense disambiguation (WSD): given a list of senses for target lemma $w$, the task is to pick the best-fitting sense for a given occurrence of $w$. The list of senses is usually taken from an online dictionary or thesaurus. However, clear cut sense boundaries are sometimes hard to define, and the meaning of words depends strongly on the context in which they are used (Cruse, 2000; Hanks, 2000). Some researchers in lexical semantics have suggested that word meanings lie on a continuum between i) clear cut cases of ambiguity and ii) vagueness where clear cut boundaries do not hold (Tuggy, 1993). Certainly, it seems that a more complex representation of word sense is needed with a softer, graded representation of meaning rather than a fixed listing of senses (Cruse, 2000).

A recent annotation study ((Erk et al., 2009), hereafter GWS) marked a target word in context with graded ratings (on a scale of 1-5) on senses from WordNet (Fellbaum, 1998). Table 1 shows an example of a sentence with the target word in bold, and with the annotator judgments given to each sense. The study found that annotators made ample use of the intermediate ratings on the scale, and often gave high ratings to more than one WordNet sense for the same occurrence. It was found that the annotator ratings could not easily be transformed to categorial judgments by making more coarse-grained senses. If human word sense judgments are best viewed as graded, it makes sense to explore models of word sense that can predict graded sense assignments.

In this paper we look at the issue of graded applicability of word sense from the point of view of automatic graded word sense assignment, using the GWS graded word sense dataset. We make three primary contributions. Firstly, we propose evaluation metrics that can be used on graded word sense judgments. Some of these metrics, like Spearman's $\rho$, have been used previously (McCarthy et al., 2003; Mitchell and Lapata, 2008), but we also introduce new metrics based on the traditional precision and recall. Secondly, we investigate how two classes of models perform on the task of graded word sense assignment: on the one hand classical WSD models, on the other hand prototype-based vector space models that can be viewed as simple one-class classifiers. We study supervised models, training on traditional WSD data and evaluating against a graded scale. Thirdly, the evaluation metrics we use also provides a novel analysis of annotator performance on the GWS dataset.

\section{Related Work}

WSD has to date been a task where word senses are viewed as having clear cut boundaries. However, there are indications that word meanings do not behave in this way (Kilgarriff, 2006). Researchers in the field of WSD have acknowledged these problems but have used existing lexical resources in the hope that useful applications can be built with them. However, there is no consensus on which 


\begin{tabular}{|c|c|c|c|c|c|c|c|c|}
\hline \multirow[b]{2}{*}{ Sentence } & \multicolumn{7}{|c|}{ Senses } & \multirow[b]{2}{*}{ Annotator } \\
\hline & 1 & 2 & 3 & 4 & 5 & 6 & 7 & \\
\hline \multirow{4}{*}{$\begin{array}{l}\text { This can be justified thermodynamically in this case, and } \\
\text { this will be done in a separate paper which is being } \\
\text { prepared. }\end{array}$} & $\overline{2}$ & $\overline{3}$ & 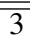 & $\overline{5}$ & $\overline{5}$ & $\overline{2}$ & $\overline{3}$ & Ann. 1 \\
\hline & 1 & 3 & 1 & 3 & 5 & 1 & 1 & Ann. 2 \\
\hline & 1 & 5 & 2 & 1 & 5 & 1 & 1 & Ann. 3 \\
\hline & 1.3 & 3.7 & 2 & 3 & 5 & 1.3 & 1.7 & Avg \\
\hline
\end{tabular}

Table 1: A sample annotation in the GWS experiment. The senses are: 1 material from cellulose 2 report 3 publication 4 medium for writing 5 scientific 6 publishing firm 7 physical object

inventory is suitable for which application, other than cross-lingual applications where the inventory can be determined from parallel data (Carpuat and $\mathrm{Wu}, 2007$; Chan et al., 2007). For monolingual applications however it is less clear whether current state-of-the-art WSD systems for tagging text with dictionary senses are able to have an impact on applications.

One way of addressing the problem of low interannotator agreement and system performance is to create an inventory that is coarse-grained enough for humans and computers to do the job reliably (Ide and Wilks, 2006; Hovy et al., 2006; Palmer et al., 2007). Such coarse-grained inventories can be produced manually from scratch (Hovy et al., 2006) or by automatically relating (McCarthy, 2006) or clustering (Navigli, 2006; Navigli et al., 2007) existing word senses. While the reduction in polysemy makes the task easier, we do not know which are the right distinctions to retain. In fact, fine-grained distinctions may be more useful than coarse-grained ones for some applications (Stokoe, 2005). Furthermore, Hanks (2000) goes further and argues that while the ability to distinguish coarse-grained senses is indeed desirable, subtler and more complex representations of word meaning are necessary for text understanding.

In this paper, instead of focusing on issues of granularity we try to predict graded judgments of word sense applicability, using a recent dataset with graded annotation (Erk et al., 2009). Our hope is that models which can mimic graded human judgments on the same task should better reflect the underlying phenomena of word meaning compared to a system that focuses on making clear cut distinctions. Also, we hope that such models might prove more useful in applications. There is one existing study of graded sense assignment (Ramakrishnan et al., 2004). It tries to estimate a probability distribution over senses by converting all of WordNet into a huge Bayesian Network, and reports improvements in a Question
Answering task. However, it does not test its prediction against human annotator data.

We concentrate on supervised models in this paper since they generally perform better than their unsupervised or knowledge-based counterparts (Navigli, 2009). We compare them against a baseline model which simply uses the training data to obtain a probability distribution over senses regardless of context, since marginal distributions are highly skewed making a prior distribution very informative (Chan and $\mathrm{Ng}$, 2005; Lapata and Brew, 2004).

Along with standard WSD models, we evaluate vector space models that use the training data to locate a word sense in semantic space. Word sense and vector space models have been related in two ways. On the one hand, vector space models have been used for inducing word senses (Schütze, 1998; Pantel and Lin, 2002). The different meanings of a word are obtained by clustering vectors. The clusters must then be mapped to an inventory if a standard WSD dataset is used for evaluation. In contrast, we use sense tagged training data with the aim of building models of given word senses, rather than clustering occurrences into word senses. The second way in which word sense and vector space models have been related is to assign disambiguated feature vectors to WordNet concepts (Pantel, 2005; Patwardhan and Pedersen, 2006). However those works do not use sense-tagged data and are not aimed at WSD, rather the applications are to insert new concepts into an ontology and to measure the relatedness of concepts.

We are not concerned in this paper with arguing for or against any particular sense inventory. WordNet has been criticized for being overly finegrained (Navigli et al., 2007; Ide and Wilks, 2006), we are using it here because it is the sense inventory used by Erk et al. (2009). That annotation study used it because it is sufficiently fine-grained to allow for the examination of subtle distinctions between usages and because it is publicly available 


\begin{tabular}{|c|c|c|c|}
\hline \multirow{2}{*}{$\begin{array}{l}\text { lemma } \\
\text { (PoS) }\end{array}$} & \multirow{2}{*}{$\begin{array}{c}\# \\
\text { senses }\end{array}$} & \multicolumn{2}{|c|}{ \# training } \\
\hline & & SemCor & SE-3 \\
\hline add (v) & 6 & 171 & 238 \\
\hline argument (n) & 7 & 14 & 195 \\
\hline ask (v) & 7 & 386 & 236 \\
\hline different (a) & 5 & 106 & 73 \\
\hline important (a) & 5 & 125 & 11 \\
\hline interest (n) & 7 & 111 & 160 \\
\hline paper (n) & 7 & 46 & 207 \\
\hline win $(v)$ & 4 & 88 & 53 \\
\hline \multicolumn{2}{|l|}{ total training $\mathrm{s}$} & 1047 & 1173 \\
\hline
\end{tabular}

Table 2: Lemmas used in this study

with various sense-tagged datasets (e.g. (Miller et al., 1993; Mihalcea et al., 2004)) for comparison.

\section{Data}

In this paper, we use a subset of the GWS dataset (Erk et al., 2009) where three annotators supplied ordinal judgments of the applicability of WordNet (v3.0) senses on a 5 point scale: 1 completely different, 2 - mostly different, 3 - similar, 4 - very similar and 5 - identical. Table 1 shows a sample annotation. The sentences that we use from the GWS dataset were originally extracted from the English SENSEVAL-3 lexical sample task (Mihalcea et al., 2004) (hereafter SE-3) and SemCor (Miller et al., 1993). ${ }^{1}$ For 8 lemmas, 25 sentences were randomly sampled from SemCor and 25 randomly sampled from SE-3, giving a total of 50 sentences per lemma. The lemmas, their PoS and number of senses from WordNet are shown in table 2.

The annotation study found that annotators made ample use of the intermediate levels of applicability (2-4), and they often gave positive ratings (3-5) to more than one sense for a single occurrence. The example in Table 1 is one such case. An analysis of the annotator ratings found that they could not easily be explained in categorial terms by making more coarse-grained senses because senses that were not positively correlated often had high ratings for the same instance.

The GWS dataset contains a sequence of judgments for each occurrence of a target word in a sentence context: one judgment for each WordNet sense of the target word. To obtain a single judgment for each sense in each sentence we use the average judgment from the three annotators. As models typically assign values between

\footnotetext{
${ }^{1}$ The GWS data also contains data from the English Lexical Substitution Task (McCarthy and Navigli, 2007) but we do not use that portion of the data for these experiments.
}

0 and 1, we normalize the annotator judgments from the GWS dataset to fall into the same range by using normalized_judgment $=$ (judgment 1.0)/4.0. This maps an original judgment of 5 to a normalized judgment of 1.0, it maps an original 1 to 0.0 , and intermediate judgments are mapped accordingly.

As the GWS dataset is too small to accommodate both training and testing of a supervised model, we use all the data from GWS for testing our models, and train our models on traditional word sense annotation data. We use as training data all sentences from SemCor and the training portion of SE-3 that are not included in GWS. The quantity of training data available is shown in the last two columns of table 2 .

\section{Evaluating Graded Word Sense Assignment}

This section discusses measures for evaluating system performance for the case where gold judgments are graded rather than categorial.

Correlation. The standard method for comparing a list of graded gold judgments to a list of graded predicted judgments is by testing for correlation. In our case, as we cannot assume a normal distribution of the judgments, a non-parametric test such as Spearman's $\rho$ will be appropriate. Spearman's $\rho$ uses the formula of Pearson's coefficient, defined as

$$
\rho(X, Y)=\frac{\operatorname{cov}(X, Y)}{\sigma_{X} \sigma_{Y}}
$$

Pearson's coefficient computes the correlation of two random variables $X$ and $Y$ as their covariance divided by the product of their standard deviations. In the computation of Spearman's $\rho$, values are transformed to rankings before the formula is applied. ${ }^{2}$ As Spearman's $\rho$ compares the rankings of two sets of judgments, it abstracts from the absolute values of the judgments. It is useful to have a measure that abstracts from absolute values of judgments and magnitude of difference because the GWS dataset contains annotator judgments on a fixed scale, and it is quite possible that human judges will differ in how they use such a scale.

Each judgment in the gold-standard can be represented as a 4-tuple 〈lemma, sense_no, sentence_no, gold_judgment $\rangle$. For example, $\langle$ add.v,

\footnotetext{
${ }^{2}$ Mitchell and Lapata (2008) note that Spearman's $\rho$ tends to yield smaller coefficients than its parametric counterparts such as Pearson's coefficient.
} 
$1,1,0.8\rangle$ is the first sentence for target add.v, first WordNet sense, with a (normalized) judgment of 0.8 . Likewise, each prediction by the model can be represented as a 4-tuple $\langle$ lemma, sense_no, sentence_no, predicted_judgment $\rangle$. We write $G$ for the set of gold tuples, $A$ for the set of assigned tuples, $L$ for the set of lemmas, $S_{\ell}$ for the set of sense numbers that exist for lemma $\ell$, and $T$ for the set of sentence numbers (there are 50 sentences for each lemma). We write $\left.G\right|_{l e m m a=\ell}$ for the gold set restricted to those tuples with lemma $\ell$, and analogously for other set restrictions and for $A$. There are several possibilities for measuring correlation:

by lemma: for each lemma $\ell \in L$, compute correlation between $\left.G\right|_{\text {lemma= } \ell}$ and $\left.A\right|_{\text {lemma }=\ell}$

by lemma+sense: for each lemma $\ell$ and each sense number $i \in S_{\ell}$, compute correlation between $\left.G\right|_{\text {lemma }}$, ,senseno= $i$ and $\left.A\right|_{\text {lemma }=\ell, \text { senseno }=i}$

by lemma+sentence: for each lemma $\ell$ and sentence number $t \in T$, compute correlation between $\left.G\right|_{\text {lemma }=\ell, \text { sentence }=t}$ and $\left.A\right|_{\text {lemma }=\ell, \text { sentence }=t}$

Comparison by lemma tests for the consistent use of judgments for the same target lemma. A comparison by lemma+sense ranks all occurrences of the same target lemma by how strongly they evoke a given word sense. A comparison by lemma+sentence ranks different senses by how strongly they apply to a given target lemma occurrence. In reporting correlation by lemma (by lemma+sense, by lemma+sentence), we average over all lemmas (lemma+sense, lemma+sentence combinations), and we report the percentage of lemmas (combinations) for which the correlation was significant. We report averaged correlation by lemma rather than one overall correlation over all judgments in order not to give more weight to lemmas with more senses.

Divergence. Another possibility for measuring the performance of a graded sense assignment model is to use Jensen/Shannon divergence (J/S), which is a symmetric version of Kullback/Leibler divergence. Given two probability distributions $p, q$, the Kullback/Leibler divergence of $q$ from $p$ is

$$
D(p \| q)=\sum_{x} p(x) \log \frac{p(x)}{q(x)}
$$

and their $\mathrm{J} / \mathrm{S}$ is

$$
J S(p, q)=\frac{1}{2}\left(D\left(p \| \frac{p+q}{2}\right)+D\left(q \| \frac{p+q}{2}\right)\right.
$$

We will use $\mathrm{J} / \mathrm{S}$ for an evaluation by lemma+sentence: for each lemma $\ell \in L$ and sentence number $t \in T$, we normalize $\left.G\right|_{\text {lemma }=\ell \text {,sentence }=t}$, the set of judgments for senses of $\ell$ in $t$, by the sum of sense judgments for $\ell$ and $t$. We do the same for $\left.A\right|_{\text {lemma }=\ell \text {,sentence }=t}$. Then we compute J/S. In doing so, we are not trying to interpret $\left.G\right|_{\text {lemma }=\ell \text {, sentence }=t}$ as some kind of probability distribution over senses, rather we use $\mathrm{J} / \mathrm{S}$ as a measure that abstracts from absolute judgments but not from the magnitude of differences between judgments.

Precision and Recall. We have discussed a measure that abstracts from both absolute judgments and magnitude of differences (Spearman's $\rho$ ), and a measure that abstracts from absolute judgments but not the magnitude of differences $(\mathrm{J} / \mathrm{S})$. What is still missing is a measure that tests to what degree a model conforms to the absolute judgments given by the human annotators.

To obtain a measure for performance in predicting absolute gold judgments, we generalize precision and recall. In the categorial case, precision is defined as $P=\frac{\text { true positives }}{\text { true positives }+ \text { false positives }}$, true positives divided by system-assigned positives, and recall is $R=\frac{\text { true positives }}{\text { true positives+false negatives }}$, true positives divided by gold positives. Writing $\operatorname{gold}_{\ell, i, t}$ for the judgment $j$ associated with lemma $\ell$ and sense number $i$ for sentence $t$ in the gold data (i.e., $\langle\ell, i, t, j\rangle \in G)$, and analogously assigned $\mathrm{d}_{\ell, i, t}$, we extend precision and recall to the graded case as follows:

$$
P_{\ell}=\frac{\sum_{i \in S_{\ell}, t \in T} \min \left(\operatorname{gold}_{\ell, i, t}, \operatorname{assigned}_{\ell, i, t}\right)}{\sum_{i \in S_{\ell}, t \in T} \operatorname{assigned}_{\ell, i, t}}
$$

and

$$
R_{\ell}=\frac{\sum_{i \in S_{\ell}, t \in T} \min \left(\operatorname{gold}_{\ell, i, t}, \operatorname{assigned}_{\ell, i, t}\right)}{\sum_{i \in S_{\ell}, t \in T} \operatorname{gold}_{\ell, i, t}}
$$

where $\ell$ is a lemma. We compute precision and recall by lemma, then macro-average them in order not to give more weight to lemmas that have more senses. The formula for F-score as the harmonic mean of precision and recall remains unchanged: $F=2 P R /(P+R)$.

If the data is categorial, the graded precision and recall measures coincide with "classical" precision 
$\mathrm{Cx} / 2$ until, IN, soft, JJ, remaining, VBG, ingredient, NNS

$\overline{\mathrm{Cx} / 50}$ for, IN, sweet-sour, NN, sauce, $\mathrm{NN}, \ldots$, to, TO, a, DT, boil, NN

Ch OA, OA/ingredient/NNS

Table 3: Sample features for add in BNC occurrence For sweet-sour sauce, cook onion in oil until soft. Add remaining ingredients and bring to a boil. $\mathrm{Cx} / 2(\mathrm{Cx} / 50)$ : context of size 2 (size 50) either side of the target. Ch: children of target.

and recall, which can be seen as follows. Graded sense assignment is represented by assigning each sense a score between 0.0 and 1.0. The categorial case can be represented in the same way, the difference being that one single sense will receive a score of 1.0 while all other senses get a score of 0.0. With this representation for categorial sense assignment, consider a fixed token $t$ of lemma $\ell$. $\sum_{i \in S_{\ell}} \min \left(\operatorname{assigned}_{\ell, i, t}, \operatorname{gold}_{\ell, i, t}\right)$ will be 1 if the assigned sense is the gold sense, and 0 otherwise.

\section{Models for Graded Word Sense Assignment}

In this section we discuss the computational models for graded word sense that are tested in this paper.

Single-best-sense WSD. The first model that we test is a standard WSD model that assigns, to each test occurrence of a target word, a single bestfitting word sense. The system thus attributes a confidence score of 1 to the assigned sense and a confidence score of 0 for all other senses for that sentence. We refer to it as WSD/single. The model uses standard features: lemma and part of speech in a narrow context window ( 2 words either side) and a wide context window (50 words either side), as well as dependency labels leading to parent, children, and siblings of the target word, and lemmas and part of speech of parent, child, and sibling nodes. Table 3 shows sample model features for an occurrence of add in the British National Corpus (BNC) (Leech, 1992). The model uses a maximum entropy learner ${ }^{3}$, training one binary classifier per sense. (With n-ary classifiers, the model's performance is slightly worse.) The model is thus not highly optimized, but fairly standard.

WSD confidence level as judgment. Our second model is the same WSD system as above, but we

\footnotetext{
${ }^{3}$ http://maxent. sourceforge.net/
}

use it to predict a judgment for each sense of a target occurrence, taking the confidence level returned by each sense-specific binary classifier as the predicted judgment. We refer to this model as WSD/conf.

Word senses as points in semantic space. The results of the GWS annotation study raise the question of how word senses are best conceptualized, given that annotators assigned graded judgments of applicability of word senses, and given that they often combined high judgments for multiple word senses. One way of modeling these findings is to view word senses as prototypes, where some uses of a word will be typical examples of a given sense, for some uses the sense will clearly not apply, and to some uses the sense will be borderline applicable.

We use a very simple model of word senses as prototypes, representing them as points in a semantic space. Graded sense applicability judgments can then be modeled using vector similarity. The dimensions of the vector space are the features of the WSD system above (including dimensions like Cx2/until, $C x 2 / I N, C h / O A /$ ingredient/NNS for the example in Table 3), and the coordinates are raw feature counts. We compute a single vector for each sense $s$, the centroid of all training occurrences that have been labeled with $s$. The predicted judgment for a test sentence and sense $s$ is then the similarity of the sentence's vector to the centroid vector for $s$, computed using cosine. We call this model Prototype. Like instance-based learners (Daelemans and den Bosch, 2005), the Prototype model measures the distance between feature vectors in space. Unlike instance-based learners, it only uses data from a single category for training.

As it is to be expected that the vectors in this space will be very sparse, we also test a variant of the Prototype model with Schütze-style secondorder vectors (Schütze, 1998), called Prototype/2. Given a (first-order) feature vector, we compute a second-order vector as the centroid of vectors for all lemma features (omitting stopwords) in the first-order vector. For the feature vector in Table 3 , this is the centroid of vectors sweet-sour, sauce, $\ldots$, boil. We compute the vectors sweet $\vec{t}$-sour etc. as dependency vectors (Padó and Lapata, 2007) ${ }^{4}$ over a Minipar parse (Lin, 1993) of the BNC.

\footnotetext{
${ }^{4}$ We use the DV package, http: //www. nlpado. de/ s sebastian/dv.html, to compute the vector space.
} 
We transform raw co-occurrence counts in the BNC-based vectors using pointwise mutual information (PMI), a common transformation function (Mitchell and Lapata, 2008). ${ }^{5}$

Another way of motivating the use of vector space models of word sense is by noting that we are trying to predict graded sense assignment by training on traditional word sense annotated data, where each target word occurrence is typically marked with a single word sense. Traditional word sense annotation, when used to predict GWS judgments, will contain spurious negative data: suppose a human annotator is annotating an occurrence of target word $t$ and views senses $s_{1}, s_{2}$ and $s_{3}$ as somewhat applicable, with sense $s_{1}$ applying most clearly. Then if the annotation guidelines ask for the best-fitting sense, the annotator should only assign $s_{1}$. The occurrence is recorded as having sense $s_{1}$, but not senses $s_{2}$ and $s_{3}$. This, then, constitutes spurious negative data for senses $s_{2}$ and $s_{3}$. The simple vector space model of word sense that we use implements a radical solution to this problem of spurious negative data: it only uses positive data for a single sense, thus forgoing competition between categories. It is to be expected that not using competition between categories will hurt the vector space model's performance, but this design gives us the chance to compare two model classes that use opposing strategies with respect to spurious negative data: the WSD models fully trust the negative data, while the vector space models ignore it.

\section{Experiments}

This section reports on experiments for the task of graded word sense assignment. As data, we use the GWS dataset described in Sec. 3. We test the models discussed in Sec. 5, evaluating with the methods described in Sec. 4.

To put the models' performance into perspective, we first consider the human performance on the task, shown in Table 4. The first three lines of the table show the performance of each annotator evaluated against the average of the other two. The fourth line averages over the previous three lines to provide an average human ceiling for the task. In the correlation of rankings by lemma, correlation is statistically significant for all lemmas at

\footnotetext{
${ }^{5}$ We also tested PMI transformation for the first-order vectors, but will not report the results here as they were worse across the board than without PMI.
}

$p \leq 0.01$. For correlation by lemma+sense and by lemma+sentence, the percentage of pairs with significant correlation is lower: 73.6 of lemma/sense pairs and 29.0 of lemma/sentence pairs reach significance at $p \leq 0.05$. For $p \leq 0.01$, the percentage is 58.3 and 12.2 , respectively. The higher $\rho$ but lower proportion of significant values for lemma+sentence pairs compared to lemma+sense is due to the fact that there are far fewer datapoints (sample size) for each calculation of $\rho$ (\#senses for lemma+sentence vs 50 sentences for lemma+sense).

At $0.131, \mathrm{~J} / \mathrm{S}$ for Annotator 1 is considerably lower than for Annotators 2 and 3. ${ }^{6}$ In terms of precision and recall, Annotator 1 again differs from the other two. At 87.5, her recall is higher than her precision (50.6), while the other annotators have considerably higher precision (75.5 and 82.4) than recall (62.4 and 52.3). This indicates that Annotator 1 tended to assign higher ratings throughout, an impression that is confirmed by Table 6 . The left two columns show average ratings for each annotator over all senses of all tokens (normalized to values between 0.0 and 1.0 as described in Sec. 3). The three annotators differ widely in their average ratings, which range from 0.285 for Ann.3 to 0.540 for Ann.1.

Standard WSD. We tested the performance of the WSD/single model on a standard WSD task, using the same training and testing data as in our subsequent experiments, as described in section 3. ${ }^{7}$ The model's accuracy when trained and tested on SemCor was A=77.0\%, with a most frequent sense baseline of $63.5 \%$. When trained and tested on SE- 3 , the model achieved $\mathrm{A}=53.0 \%$ against a baseline of $44.0 \%$. When trained and tested on SemCor plus SE-3, the model reached an accuracy $58.2 \%$, with a baseline of $56.0 \%$. So on the combined dataset, the baseline is the average of the baselines on the individual datasets, while the model's performance falls below the average performance on the individual datasets.

WSD models for graded sense assignment. Table 5 shows the performance of different models in the task of graded word sense assignment. The first line in Table 5 lists results for the maximum entropy model when used to assign a single best sense. The second line lists the results for

\footnotetext{
${ }^{6}$ Low J/S implies a closer agreement between two sets of judgments.

${ }^{7}$ Note that this constitutes less training data than in the SE-3 task.
} 


\begin{tabular}{|c|c|c|c|c|c|c|c|c|c|c|c|c|c|}
\hline \multirow[b]{2}{*}{ Ann } & \multirow[b]{2}{*}{$\rho$} & \multicolumn{2}{|c|}{ y lemma } & \multicolumn{3}{|c|}{ by lemma+sense } & \multicolumn{3}{|c|}{ by lemma+sentence } & \multirow[b]{2}{*}{$\mathrm{J} / \mathrm{S}$} & \multirow[b]{2}{*}{$\mathrm{P}$} & \multirow[b]{2}{*}{$\mathrm{R}$} & \multirow[b]{2}{*}{$\mathrm{F}$} \\
\hline & & * & $* *$ & $\rho$ & * & $* *$ & $\rho$ & * & $* *$ & & & & \\
\hline$\overline{\text { Ann.1 }}$ & $\overline{0.517}$ & $\overline{100.0}$ & $\overline{100.0}$ & $\overline{0.407}$ & $\overline{75.0}$ & $\overline{58.3}$ & 0.482 & 27.3 & 11.5 & 0.131 & 50.6 & 87.5 & 64.1 \\
\hline Ann.2 & 0.587 & 100.0 & 100.0 & 0.403 & 68.8 & 58.3 & 0.612 & 38.1 & 17.2 & 0.153 & 75.5 & 62.4 & 68.3 \\
\hline Ann.3 & 0.528 & 100.0 & 100.0 & 0.41 & 77.1 & 58.3 & 0.51 & 21.8 & 7.8 & 0.165 & 82.4 & 52.3 & 64.0 \\
\hline$\overline{\mathrm{Avg}}$ & 0.544 & 100.0 & 100.0 & 0.407 & 73.6 & 58.3 & 0.535 & 29.0 & 12.2 & 0.149 & 69.5 & 67.4 & 65.5 \\
\hline
\end{tabular}

Table 4: Human ceiling: one annotator vs. average of the other two annotators. $*, * *$ : percentage significant at $p \leq 0.05, p \leq 0.01$. Avg: average annotator performance

\begin{tabular}{|c|c|c|c|c|c|c|c|c|c|c|c|c|c|}
\hline \multirow[b]{2}{*}{ Model } & \multicolumn{3}{|c|}{ by lemma } & \multicolumn{3}{|c|}{ by lemma+sense } & \multicolumn{3}{|c|}{ by lemma+sentence } & \multirow[b]{2}{*}{$\mathrm{J} / \mathrm{S}$} & \multirow[b]{2}{*}{$\mathrm{P}$} & \multirow[b]{2}{*}{$\mathrm{R}$} & \multirow[b]{2}{*}{$\mathrm{F}$} \\
\hline & $\rho$ & $*$ & $* *$ & $\rho$ & $*$ & $* *$ & $\rho$ & $*$ & $* *$ & & & & \\
\hline$\overline{\overline{\text { WSD/single }}}$ & 0.267 & 87.5 & $\overline{75.0}$ & 0.053 & $\overline{6.3}$ & $\overline{4.2}$ & 0.28 & 2.8 & $\overline{1.8}$ & 0.39 & 58.7 & 25.5 & 35.5 \\
\hline WSD/conf & 0.396 & 87.5 & 87.5 & 0.177 & 33.3 & 18.8 & 0.401 & 10.8 & 3.0 & 0.164 & 81.8 & 37.1 & 51.0 \\
\hline Prototype & 0.245 & 62.5 & 62.5 & 0.053 & 20.8 & 8.3 & 0.396 & 15.3 & 2.5 & 0.173 & 58.4 & 78.3 & 66.9 \\
\hline Prototype/2 & 0.292 & 87.5 & 87.5 & 0.086 & 14.6 & 4.2 & 0.478 & 22.8 & 7.5 & 0.164 & 68.2 & 63.3 & 65.7 \\
\hline Prototype/N & 0.396 & 100.0 & 100.0 & 0.137 & 22.9 & 14.6 & 0.396 & 15.3 & 2.5 & 0.173 & 82.2 & 29.9 & 43.9 \\
\hline Prototype/2N & 0.465 & 100.0 & 100.0 & 0.168 & 29.8 & 23.4 & 0.478 & 22.8 & 7.5 & 0.164 & 82.6 & 30.9 & 45.0 \\
\hline baseline & 0.338 & 87.5 & 87.5 & 0.0 & 0.0 & 0.0 & 0.355 & 10.3 & 3.0 & 0.167 & 79.9 & 34.5 & 48.2 \\
\hline
\end{tabular}

Table 5: Evaluation: computational models, and baseline. $*, * *$ : percentage significant at $p \leq 0.05$, $p \leq 0.01$

the same maximum entropy model when classifier confidence is used as predicted judgment. The last line shows the baseline, an adaptation of the most frequent sense baseline to the graded case. For this baseline, we computed the relative frequency of each sense in the training corpus and used this relative frequency as the prediction for each test sentence and sense combination. The WSD/single model remains below the baseline in all evaluations except correlation by lemma+sense, where no rank-based correlation could be computed for the baseline because it always assigns the same judgment for a given sense. WSD/conf shows a performance slightly above the baseline in all evaluation measures. Table 6 lists average ratings, averaged over all lemmas, senses, and occurrences, for each model in the two right-hand columns.

Prototype models. Lines 3-6 in Tables 5 and 6 show results for Prototype variants. While each Prototype and Prototype/2 model only sees positive data annotated for a single sense, the variants with $/ N$ (lines 5 and 6 ) make very limited use of information coming from all senses of a given lemma. They normalize judgments for each sentence, with

$$
\operatorname{assigned}_{\ell, i, t}^{\text {norm }}=\frac{\text { assigned }_{\ell, i, t}}{\sum_{j \in S_{\ell}} \operatorname{assigned}_{\ell, j, t}}
$$

Line 3 evaluates the Prototype model with firstorder vectors. Its correlation with the gold data is somewhat lower than that of WSD/conf in almost all cases. ${ }^{8}$ The Prototype model deviates strongly

\footnotetext{
${ }^{8}$ The reason why the average $\rho$ for correlation by
}

from both $W S D /$ conf and baseline in having a very good recall, at 78.3, with lower precision at 58.4, for an overall F-score that is 16 points higher than that of WSD/conf. Both Prototype and Prototype/2 have average ratings (Table 6) far above those of the WSD models and of the $/ N$ variants. The second-order vector model Prototype/2 has relatively low correlation by lemma+sense, while correlation by lemma+sentence shows the best performance of all models (along with Prototype/2N). Its correlation by lemma+sentence is similar to the lowest correlation by lemma+sentence achieved by a human annotator. In terms of $\mathrm{J} / \mathrm{S}$, this model also shows the best performance along with WSD/conf and Prototype/2N. Both $/ N$ variants achieve very high correlation by lemma. Correlation by lemma+sense for the $/ N$ models is between those of Prototype and WSD/conf. The correlation by lemma+sentence is the same with or without normalization, as normalization does not change the ranking of senses of an individual sentence. While Prototype has higher recall than precision, normalization turns it into a model with even higher precision than WSD/conf but even lower recall.

\section{Discussion}

Human performance. The evaluation of human annotators in Table 4 provides a novel analysis of the GWS dataset over and above that by Erk et al.

lemma+sense is the same for Prototype and WSD/single while the significance percentage differs greatly is that the Prototype shows negative correlation for some of the senses. 


\begin{tabular}{c|c||c|c} 
Ann. & avg & Model & avg \\
\hline \hline Ann.1 & 0.540 & WSD/single & 0.163 \\
Ann.2 & 0.345 & WSD/conf & 0.173 \\
\cline { 3 - 4 } Ann.3 & 0.285 & Prototype & 0.558 \\
& & Prototype/N & 0.143 \\
& & Prototype/2 & 0.375 \\
& & Prototype/2N & 0.143 \\
\cline { 3 - 4 } & & baseline & 0.167
\end{tabular}

Table 6: Average judgment for individual annotators (transformed) and average rating for models

(2009). Human annotators show very strong correlation of their rankings by lemma. They also had strong agreement on rankings by lemma+sense, which ranks occurrences of a lemma by how strongly they evoke a given sense. The relatively low precision and recall in Table 4 confirm that different annotators use the 5-point scale in different ways. A comparison of precision and recall between the annotators reflects the fact that Annotator 1 tended to give considerably higher ratings than the other two, which is also apparent in the average ratings in Table 6. Given the relatively low F-score achieved by human annotators, judgments by additional annotators could make the GWS dataset more useful, in that the average judgments would not be influenced so strongly by idiosyncrasies in the use of the 5-point scale. (Psycholinguistic experiments using fixed scales typically elicit judgments from 10 or more participants per item.)

Evaluation measures. Given the degree of differences in the absolute values of the human annotator judgments (Table 4), a rank-based evaluation of graded sense assignment models, complemented by $\mathrm{J} / \mathrm{S}$ to evaluate the magnitude of differences between ratings, seems most appropriate to the data. Rankings by lemma+sense and by lemma+sentence are especially interesting for their potential use in systems that might use graded sense assignment as part of a larger pipeline. Still, the new graded precision and recall measures allow for a more fine-grained analysis of the performance of models, showing fundamental differences in the behavior of WSD/conf and the Prototype model. Graded precision and recall could become even more informative measures with a gold set containing judgments of more annotators, since then the absolute gold judgments would be more reliable.

Standard WSD models and vector space models. The results in Table 5 reflect the compromise between the advantage of having competition between categories and the disadvantage of spurious negative data: WSD/conf, Prototype/N and Prototype/ $2 \mathrm{~N}$ achieve the highest correlation by lemma, and high precision, while Prototype has much better recall for an overall higher F-score. However, as Table 6 shows, Prototype tends to assign high ratings across the board, leading to high recall. The much lower average ratings of the $/ N$ models explain their higher precision and lower recall: they overshoot less and undershoot more. The improvement in correlation for the $/ N$ models also indicates that Prototype assigns some sentences high ratings for all senses, impacting rankings by lemma and by lemma+sense.

The comparison of Prototype and Prototype/2 gives us a chance to study effects of feature sparseness. Prototype/2, using second-order vectors that should be much less sparse, yields better rankings than Prototype. The average ratings of model Prototype/2 (Table 6) are lower than those of Prototype (and closer to human average ratings), resulting in higher precision and lower recall. One possible reason for the high average ratings of Prototype is that in sparser (and shorter) vectors, matches in dimensions for high-frequency, relatively uninformative context items have greater impact.

It is interesting to see that WSD/conf performs slightly above the sense frequency baseline in all evaluations, since this is a very familiar picture from standard WSD.

Prototype/ $2 N$ shows the overall most favorable performance in terms of correlation as it i) pays minimal attention to the negative data ii) uses normalization to avoid overshooting and iii) compensates for sparse data by using second order vectors. For J/S, WSD/conf, Prototype/2, Prototype/2N and the sense frequency baseline just outperform the score of the lowest-scoring of the three annotators. In terms of F-score, Prototype shows results very close to human performance. Interestingly, the Prototype model resembles Annotator 1 in its precision and recall, while WSD/conf more resembles Annotators 2 and 3. None of the models come close to human performance in ranking by lemma+sense, which requires an identification of the "typical" occurrence of a given sense. The low ratings in correlation by lemma+sense indicate that the models might be limited by the lack of training data for many of the rarer senses. In fu- 
ture work, we will test how the frequency of senses in the training data affects the different models.

\section{Conclusion}

In this paper we have done a first study on modeling graded annotator judgments on sense applicability. We have discussed evaluation measures for models of graded sense assignment, including new extensions of precision and recall to the graded case. A combination of rank-based correlation at the level of lemmas, senses, and sentences, Jensen/Shannon divergence, and precision and recall provided a nuanced picture of the strengths and weaknesses of different models. We have tested two types of models: on the one hand a standard binary WSD model using classifier confidence as predicted judgments, and on the other hand several vector space models which compute a prototype vector for each sense in semantic space. These two types of model differ strongly in their behavior. The WSD model shows a similar behavior as the baseline, with high precision but low recall, while the unnormalized version of the vector space model has higher recall at lower precision. The results show both the benefits of having competition between categories, for improved rankbased correlation and precision, and the problem of spurious negative data in the training set arising from the best-sense methodology.

The last two correlation measures, by lemma+sense and by lemma+sentence, yield maybe the most insight into the question of the usability of a computational model for graded word sense assignment: a graded word sense assignment model that is a component of a larger system could provide useful sense information either by ranking occurrences by how strongly they evoke a sense, or by ranking senses by how strongly they apply to a given occurrence. There is room for improvement however as system performance is well below that of humans. In the future we plan to investigate features that are more informative for making graded judgments. Second, the vector space model we used was very simple; it might be worthwhile to test more sophisticated one-class classifiers (Marsland, 2003; Schölkopf et al., 2000).

Acknowledgments. We acknowledge support from the UK Royal Society for a Dorothy Hodgkin Fellowship to the second author.

\section{References}

M. Carpuat and D. Wu. 2007. Improving statistical machine translation using word sense disambiguation. In Proceedings of EMNLP-CoNLL 2007, pages 61-72, Prague, Czech Republic, June. Association for Computational Linguistics.

Y. S. Chan and H. T. Ng. 2005. Word sense disambiguation with distribution estimation. In Proceedings of IJCAI 2005, pages 1010-1015, Edinburgh, Scotland.

Y. S. Chan, H. T. Ng, and D. Chiang. 2007. Word sense disambiguation improves statistical machine translation. In Proceedings of ACL'07, Prague, Czech Republic, June.

D. A. Cruse. 2000. Aspects of the microstructure of word meanings. In Y. Ravin and C. Leacock, editors, Polysemy: Theoretical and Computational Approaches, pages 30-51. OUP, Oxford, UK.

W. Daelemans and A. Van den Bosch. 2005. MemoryBased Language Processing. Cambridge University Press, Cambridge, UK.

K. Erk, D. McCarthy, and N. Gaylord. 2009. Investigations on word senses and word usages. In Proceedings of ACL-09, Singapore.

C. Fellbaum, editor. 1998. WordNet, An Electronic Lexical Database. The MIT Press, Cambridge, MA.

P. Hanks. 2000. Do word meanings exist? Computers and the Humanities, 34(1-2):205-215(11).

E. Hovy, M. Marcus, M. Palmer, L. Ramshaw, and R. Weischedel. 2006. Ontonotes: The 90\% solution. In Proceedings of the HLT-NAACL 2006 workshop on Learning word meaning from non-linguistic data, New York City, USA. Association for Computational Linguistics.

N. Ide and Y. Wilks. 2006. Making sense about sense. In E. Agirre and P. Edmonds, editors, Word Sense Disambiguation, Algorithms and Applications, pages 47-73. Springer.

A. Kilgarriff. 2006. Word senses. In E. Agirre and P. Edmonds, editors, Word Sense Disambiguation, Algorithms and Applications, pages 29-46. Springer.

M. Lapata and C. Brew. 2004. Verb class disambiguation using informative priors. Computational Linguistics, 30(1):45-75.

G. Leech. 1992. 100 million words of English: the British National Corpus. Language Research, 28(1):1-13.

D. Lin. 1993. Principle-based parsing without overgeneration. In Proceedings of ACL'93, Columbus, Ohio, USA. 
S. Marsland. 2003. Novelty detection in learning systems. Neural computing surveys, 3:157-195.

D. McCarthy and R. Navigli. 2007. SemEval-2007 task 10: English lexical substitution task. In Proceedings of SemEval-2007, pages 48-53, Prague, Czech Republic.

D. McCarthy, B. Keller, and J. Carroll. 2003. Detecting a continuum of compositionality in phrasal verbs. In Proceedings of the ACL 03 Workshop: Multiword expressions: analysis, acquisition and treatment, pages 73-80.

D. McCarthy. 2006. Relating WordNet senses for word sense disambiguation. In Proceedings of the ACL Workshop on Making Sense of Sense: Bringing Psycholinguistics and Computational Linguistics Together, pages 17-24, Trento, Italy.

R. Mihalcea, T. Chklovski, and A. Kilgarriff. 2004. The Senseval-3 English lexical sample task. In Proceedings of SensEval-3, Barcelona, Spain.

G. A. Miller, C. Leacock, R. Tengi, and R. T Bunker. 1993. A semantic concordance. In Proceedings of the ARPA Workshop on Human Language Technology, pages 303-308. Morgan Kaufman.

J. Mitchell and M. Lapata. 2008. Vector-based models of semantic composition. In Proceedings of ACL'08 - HLT, pages 236-244, Columbus, Ohio.

R. Navigli, K. C. Litkowski, and O. Hargraves. 2007. SemEval-2007 task 7: Coarse-grained English allwords task. In Proceedings of SemEval-2007, pages 30-35, Prague, Czech Republic.

R. Navigli. 2006. Meaningful clustering of senses helps boost word sense disambiguation performance. In Proceedings of COLING-ACL 2006, pages 105-112, Sydney, Australia.

R. Navigli. 2009. Word sense disambiguation: a survey. ACM Computing Surveys, 41(2):1-69.

S. Padó and M. Lapata. 2007. Dependency-based construction of semantic space models. Computational Linguistics, 33(2):161-199.

M. Palmer, H. Trang Dang, and C. Fellbaum. 2007. Making fine-grained and coarse-grained sense distinctions, both manually and automatically. Natural Language Engineering, 13:137-163.

P. Pantel and D. Lin. 2002. Discovering word senses from text. In Proceedings of KDD'02.

P. Pantel. 2005. Inducing ontological co-occurrence vectors. In Proceedings of ACL'05, Ann Arbor, Michigan.

S. Patwardhan and T. Pedersen. 2006. Using wordnetbased context vectors to estimate the semantic relatedness of concepts. In Proceedings of the EACL 06 Workshop: Making Sense of Sense: Bringing Psycholinguistics and Computational Linguistics Together, Trento, Italy.
G. Ramakrishnan, B.P. Prithviraj, A. Deepa, P. Bhattacharyya, and S. Chakrabarti. 2004. Soft word sense disambiguation. In Proceedings of GWC 04, Brno, Czech Republic.

B. Schölkopf, R. Williamson, A. Smola, J. ShaweTaylor, and J. Platt. 2000. Support vector method for novelty detection. Advances in neural information processing systems, 12.

H. Schütze. 1998. Automatic word sense discrimination. Computational Linguistics, 24(1).

C. Stokoe. 2005. Differentiating homonymy and polysemy in information retrieval. In Proceedings of HLT/EMNLP-05, pages 403-410, Vancouver, B.C., Canada.

D. H. Tuggy. 1993. Ambiguity, polysemy and vagueness. Cognitive linguistics, 4(2):273-290. 